\title{
Iterative Feedback Tuning of PID parameters: comparison with classical tuning rules
}

\author{
Olivier Lequin $^{\text {a }}$, Michel Gevers ${ }^{\mathrm{b}, 1}$, Magnus Mossberg ${ }^{\mathrm{d}}$, \\ Emmanuel Bosmans ${ }^{\mathrm{a}}$, Lionel Triest ${ }^{\mathrm{c}}$ \\ ${ }^{a}$ Solvay S.A., 39 rue Solvay, B-5190 Jemeppe-sur-Sambre, Belgium \\ E-mail: Olivier.Lequin@solvay.com \\ ${ }^{\mathrm{b}}$ Centre for Systems Engineering and Applied Mechanics (CESAME), Université \\ catholique de Louvain, B-1348 Louvain-la-Neuve, Belgium \\ E-mail: Gevers@csam.ucl.ac.be, Fax: +32 10472180 \\ ${ }^{\mathrm{c}}$ AWTC Europe - Automatic Transmissions, Avenue de l'Industrie, 19, B-1420 \\ Braine-l'Alleud, Belgium \\ E-mail: triest@aweurope.be, Fax: +32 23891261 \\ ${ }^{\mathrm{d}}$ Dept of Electrical Engineering, Karlstad University, SE-651 88 Karlstad, Sweden \\ E-mail: Magnus.Mossberg@kau.se
}

\begin{abstract}
We apply the Iterative Feedback Tuning (IFT) method to the tuning of PID parameters in applications where the objective is to achieve a fast response to set point changes. We compare the performance of these IFT-tuned PID controllers with the performance achieved by four classical PID tuning schemes that are widely used in industry. Our simulations show that IFT always achieves a performance that is at least as good as that of the classical PID tuning schemes, and often dramatically better: faster settling time and less overshoot. In addition, IFT is also optimal with respect to the presence of noise, whereas the other schemes are designed for noise-free conditions. The IFT method used here is a variant of the initial IFT scheme, in which no weighting is applied to the control error during a time window that corresponds to the transient response, and where the lenght of this window is progressively reduced. This method was initially proposed in (Lequin, 1997), and elaborated on in (Lequin et al., 1999).
\end{abstract}

Key words: Iterative Feedback Tuning, PID tuning, optimal control

$\overline{1 \text { Corresponding author }}$ 


\section{Introduction}

The Iterative Feedback Tuning method is a model-free technique for the optimization of the parameters of a controller of fixed structure using only signal information on the closed-loop system. The method was initially derived in (Hjalmarsson et al., 1994) and has quickly proved its efficiency in both laboratory and industrial applications: see e.g. (Hjalmarsson et al., 1995), (Lequin, 1997). A complete presentation of the theory, as well as applications to controller tuning for mechanical systems and chemical plants, can be found in (Hjalmarsson et al., 1998).

In its initial formulation, as developed in (Hjalmarsson et al., 1994), IFT was derived for any two degree of freedom linear controller $C=\left[C_{r}, C_{y}\right]$, applied to an unknown linear time-invariant system: see Figure 1 . Here $r, u$, and $y$ are the reference, the control signal and the output signal, respectively, while $v$ is an unknown disturbance.

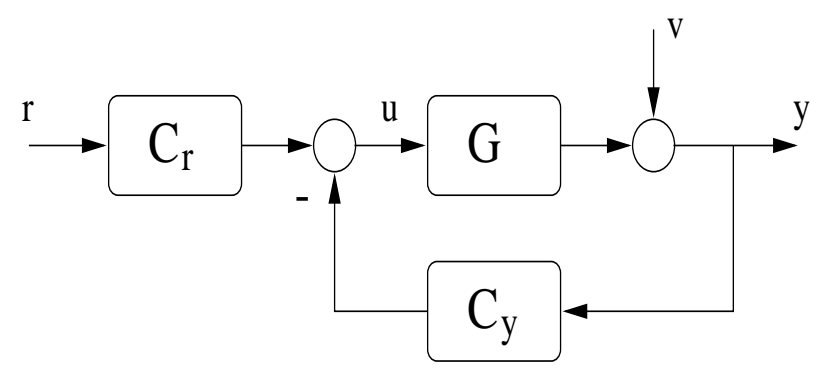

Fig. 1. Actual closed loop system

The following quadratic criterion was adopted in (Hjalmarsson et al., 1994):

$$
J(\rho)=\frac{1}{2 N} \mathrm{E}\left[\sum_{t=1}^{N}\left(L_{y} \tilde{y}_{t}(\rho)\right)^{2}+\lambda \sum_{t=1}^{N}\left(L_{u} u_{t}(\rho)\right)^{2}\right]
$$

Here $\rho$ is the vector of controller parameters to be optimized, $\tilde{y}_{t}(\rho)$ is the error between the output $y_{t}(\rho)$ of the actual system controlled by the controller $C(\rho)=\left[C_{r}(\rho), C_{y}(\rho)\right]$ and a desired output signal $y_{d}, u_{t}(\rho)$ is the control signal, $L_{y}$ and $L_{u}$ are frequency weighting filters, $\lambda$ expresses the relative importance of the penalty on the control signal versus the tracking error, $N$ is the number of data points, and E stands for expected value. The output and the control signal of the actual system are functions of the controller parameter vector $\rho$; hence the notations $y_{t}(\rho)$ and $u_{t}(\rho)$ to make this dependence transparent. The main contribution of (Hjalmarsson et al., 1994) was to show that one can compute an unbiased estimate of the gradient of this cost function without knowledge of the system, by applying a special "feedback" experiment to the actual system and by using signal information only. A (local) minimum of the cost function can then be reach by iterative computations of this gradient $\frac{\partial J}{\partial \rho_{i}}$ and the use of a stochastic approximation algorithm for the update 
of the controller parameter vector $\rho_{i}$ :

$$
\rho_{i+1}=\rho_{i}-\gamma_{i} R_{i}^{-1} \frac{\partial J}{\partial \rho}\left(\rho_{i}\right)
$$

Here $R_{i}$ is some appropriate positive definite matrix, typically a Gauss-Newton approximation of the Hessian of $J$, while $\gamma_{i}$ is a positive real scalar that determines the step size. The sequence $\left\{\gamma_{i}\right\}$ must obey some constraints for the algorithm to converge to a local minimum of the cost function $J(\rho)$ : see (Hjalmarsson et al., 1994).

In this paper we present a variant of this criterion in which the signals $\tilde{y}_{t}(\rho)$ and $u_{t}(\rho)$ (or their frequency weighted versions) are time weighted by weightings $w_{y}(t)$ and $w_{u}(t)$, respectively. This idea was initially suggested in (Lequin, 1997), where it was suggested that the criterion (1) could be replaced by the following time-weighted criterion:

$$
J_{w}(\rho)=\frac{1}{2 N} \mathrm{E}\left[\sum_{t=1}^{N} w_{y}(t)\left(L_{y} \tilde{y}_{t}(\rho)\right)^{2}+\lambda \sum_{t=1}^{N} w_{u}(t)\left(L_{u} u_{t}(\rho)\right)^{2}\right],
$$

where $w_{y}(\cdot)$ and $w_{u}(\cdot)$ are any nonnegative numbers. The flexibility offered by the time weightings $w_{y}(t)$ and $w_{u}(t)$ is that they allow one to put different weightings on different parts of the time responses.

A particularly interesting application, presented and discussed in (Lequin et al., 1999), is when zero weightings are put on the transient part of the output error in response to a step change in the reference signal, i.e. when the following criterion is used:

$$
J_{m}(\rho)=\frac{1}{2 N} \mathrm{E}\left[\sum_{t=t_{0}}^{N}\left(L_{y} \tilde{y}_{t}(\rho)\right)^{2}+\lambda \sum_{t=1}^{N}\left(L_{u} u_{t}(\rho)\right)^{2}\right] .
$$

We say in such case that a mask of length $t_{0}$ is put on the transient response of the tracking error. As explained in (Lequin et al., 1999), the motivation for the use of such masks is as follows.

One of the frequent practical uses of controller design is to tune a controller of fixed structure (for example a PID controller) in such a way that the step response of the closed-loop system has a minimal settling time with a small overshoot. The objective in such applications is to move the output of the closed-loop system quickly from one reference value to another one; however, the particular shape of the transient response from the initial reference value to the final value is of no importance, provided that it does not have large overshoots. In addition, without knowledge of the actual system (which is a major reason for using IFT) it is not known in advance how fast a settling time can be achieved for this particular system with this particular controller structure. 
By imposing the entire response of the closed-loop system through a specific choice of a desired transient response $y_{d}$, rather than just the endpoint of this transient response, the classical IFT criterion leads to controller parameters that realize a compromise between fitting the imposed transient response and fitting the new reference value, even though the user does not care about the exact shape of the transient response, as long as it is not oscillatory. Instead, by imposing a "mask" (i.e. a zero error weighting) on the transient response, the criterion (4) will tune the controller parameters in such a way as to achieve the new desired reference value without focusing on a particular pre-imposed transient response that is perhaps not naturally achieved by the closed loop system. In other words, by imposing a mask on the transient response one does not waste the available degrees of freedom in the controller parameters on the matching of a specific and entirely arbitrary transient response. Instead one can focus these parameters entirely on achieving a fast settling time. By the optimality principle, the cost achieved after the masked interval is always smaller than when no mask is used.

In (Lequin et al., 1999) the advantages of using a mask with zero weighting on the output error were demonstrated. In addition, a very simple procedure was proposed for the minimization of the settling time in response to a step change in the setpoint using the IFT scheme with a succession of masks of decreasing lengths. This procedure consists of imposing initially a rather large mask (i.e. a rather large zero-weighting time interval), and then to progressively reduce the size of the mask until oscillations start to appear in the transient response. This allows one to choose the mask of appropriate length, and hence to design the controller that achieves the smallest settling time without oscillations.

In this paper we apply this idea to the tuning of simple PID controllers. To illustrate the effectiveness of the method, we shall compare the closed loop response to a step change of a number of simulated systems, where the controller parameters are adjusted by the modified IFT procedure explained above, to the closed loop response of these same systems in which the PID parameters have been tuned by classical PID tuning rules that are widely used in industrial applications.

\section{The competitors}

In this section, we pose the problem and we briefly explain the specific PID parameter tuning methods of three different competing schemes. The PID controller structure used is one where there is no derivative action on the reference signal, as is most often the case in industrial applications. This has the advantage of avoiding large control signals when the reference signal contains 
a step change. Thus, we consider the blockdiagram of Figure 1, where

$$
\begin{aligned}
& C_{r}=K\left(1+\frac{1}{T_{i} s}\right) \\
& C_{y}=K\left(1+\frac{1}{T_{i} s}+T_{d} s\right) .
\end{aligned}
$$

The PID parameters can be collected in a vector $\rho$ :

$$
\boldsymbol{\rho}=\left[K, T_{i}, T_{d}\right]^{T}
$$

In this paper, we compare the performance of IFT for PID parameter tuning to those of three widely used PID controller tuning methods: the ZieglerNichols (ZN) tuning rules, the Internal Model Control (IMC) method and the Integral Square Error (ISE) method. One might of course question this particular selection of alternative PID tuning methods for our comparison with IFT. Clearly, we could have chosen a number of other methods, including some more recent ones. However, these three methods (and in particular ZN) are probably still the most widely used in industrial applications.

\subsection{The Ziegler-Nichols (ZN) tuning rules}

The Ziegler-Nichols tuning rules are based on what is called the "ultimate sensitivity method" (Åström and Wittenmark, 1997). It consists of determining the point where the Nyquist plot of the open loop system intersects the negative real axis. This point is obtained by connecting a purely proportional controller to the system, and by increasing the controller gain until the closed-loop system reaches the stability limit, at which oscillations occur. The oscillation period is denoted $T_{c}$ and the corresponding critical gain by $K_{c}$. The Ziegler-Nichols choice for the three PID parameters is then

$$
K=\frac{K_{c}}{1.7}, \quad T_{i}=\frac{T_{c}}{2}, \quad T_{d}=\frac{T_{c}}{8} .
$$

There exist other more recent variants of the Ziegler-Nichols tuning scheme. In our simulations, we have also implemented a recent variant proposed in (Kristiansson and Lennartson, 2000). However, the results were not significantly different for our four simulated systems than those obtained with the classical ZN scheme. 


\subsection{The Internal Model Control (IMC) method}

The principle of this method is explained on pp. 163-164 in (Åström and Hägglund, 1995). The system is approximated by a model of the form

$$
G(s)=\frac{K_{p}}{1+s T} e^{-s L} .
$$

If the actual system is unknown, the static gain $K_{p}$, the apparent time constant $T$ and the apparent deadtime $L$ are determined from an open loop step response, from which an IMC controller is then computed. The controller given by the IMC method can be interpreted as a PID controller with the following choices

$$
K=\frac{2 T+L}{2 K_{p}\left(T_{f}+L\right)}, \quad T_{i}=T+\frac{L}{2}, \quad T_{d}=\frac{T L}{2 T+L},
$$

where the design parameter $T_{f}$ corresponds to the desired closed-loop time constant. In each of our four simulations, we have optimized over this design parameter to achieve an IMC controller with minimum settling time. This optimization was performed by trial and error.

There are other variants of the IMC method, including some that can handle more general classes of model structures than (9): see e.g. (Rivera and Morari, 1987) and (Isaksson and Graebe, 1999). In order to compare methods that are of the same complexity, all our simulations in this paper are performed with an IMC method that adopts the model structure (9).

\subsection{The Integral Square Error (ISE) method}

The Integral Square Error method consists in adjusting the PID parameters in such a way as to minimize the following criterion

$$
\mathrm{ISE}=\int_{0}^{\infty} e_{t}^{2} \mathrm{~d} t=\frac{1}{2 \pi \mathrm{i}} \int_{-\mathrm{i} \infty}^{\mathrm{i} \infty} E(s) E(-s) \mathrm{d} s,
$$

where $e_{t}$ is the error between the actual response $y_{t}$ and the desired response $y_{t}^{d}$. The integral is calculated recursively using Åström's integral algorithm (Åström, 1970), and minimized with respect to the PID parameters.

\subsection{The Iterative Feedback Tuning (IFT) method}

For the simulations presented in the next section, the IFT method with mask was applied, with a mask of length $t_{0}$. Unless otherwise specified, no weighting 
was applied to the control input in the criterion. Thus, the following criterion was minimized:

$$
J(\boldsymbol{\rho})=\mathrm{E}\left\{\sum_{t=t_{0}}^{N}\left(y_{t}(\boldsymbol{\rho})-y_{t}^{d}\right)^{2}\right\} .
$$

The initial values of the PID parameters were chosen in such a way as to give an initial response that was very slow, and with no overshoot. The length $t_{0}$ of the mask was initially chosen to correspond with the settling time of this very slow response. This length was then successively reduced until oscillations appeared in the closed loop step response.

\section{Simulations}

The three classical PID tuning methods and the IFT method have been tested on the following four simulated systems. Except when otherwise stated, no noise has been added to the output; thus $v=0$ in Figure 1.

$$
\begin{aligned}
G_{1}(s) & =\frac{1}{1+20 s} e^{-5 s}, \\
G_{2}(s) & =\frac{1}{1+20 s} e^{-20 s} \\
G_{3}(s) & =\frac{1}{(1+10 s)^{8}}, \\
G_{4}(s) & =\frac{1-5 s}{(1+10 s)(1+20 s)}
\end{aligned}
$$

Note that $G_{4}$ has a nonminimum phase zero. Note also that the systems $G_{1}(s)$ and $G_{2}(s)$ are exactly of the form (9), and that the IMC controller is tailored for such systems. It also follows from Section 2.9 in (Åström and Hägglund, 1995) that the systems $G_{3}(s)$ and $G_{4}(s)$ can be approximated by the same form.

The sampling time chosen for the discretised simulations is equal to $0.01 \mathrm{~s}$. A third order Padé approximation is used for time delays. For the starting values of the PID parameters in the iterative minimization of the ISE criterion we have taken the parameters obtained by the Ziegler Nichols method. 


\subsection{Simulation 1}

The simulated system is

$$
G_{1}(s)=\frac{1}{1+20 s} e^{-5 s}
$$

The closed loop step responses obtained by the four PID tuning methods for system $G_{1}$ are shown in Figure 2. For the IMC method, the best value of the design parameter $T_{f}$ was found to be 1.3. For the IFT method, $t_{0}$ has been decreased from 70 secs to 10 secs in steps of 20 secs.
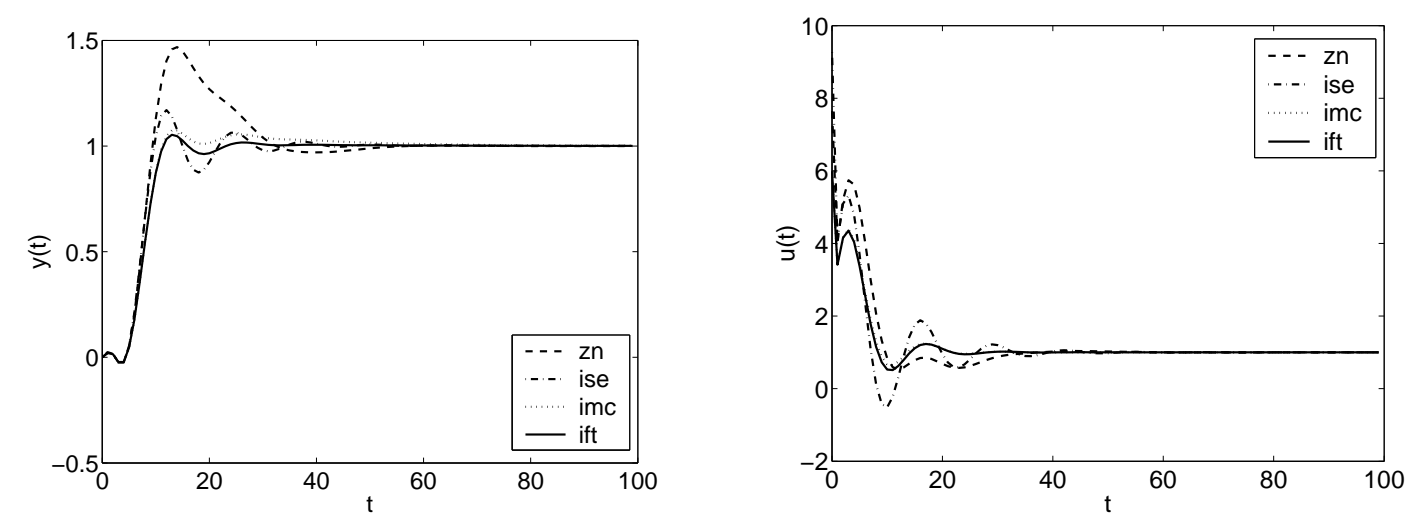

Fig. 2. Step responses (left) and corresponding input signals (right) for the closed loop systems with $G_{1}(s)$ and the PID controllers tuned with the four different methods.

Observe that the settling time of the controller tuned with IFT is significantly smaller than that obtained with the ZN and ISE tuning rules, while the overshoot with that controller is also much smaller. This is achieved with a control action that is smaller than that obtained with these other methods. The IMC method (after optimization of the design parameter $T_{f}$ ) performs much the same as the IFT controller; this method is particularly well suited for systems of the structure of $G_{1}(s)$. The worst response is achieved by the Ziegler Nichols controller.

The PID parameters produced by the four different tuning methods are given in Table 1.

Suppose now that noise is acting on the system. The IFT criterion incorporates a noise rejection as well as a tracking objective. Hence the controller parameters obtained by the IFT method will take account of the presence of noise; in fact they realize the optimal tradeoff between noise rejection and tracking. For the other tuning methods, there is no clear way of tuning the PID parameters to take account of a noise rejection objective. In addition, for 
Table 1

\begin{tabular}{|l||c|c|c|}
\hline Tuning method & $K$ & $T_{i}$ & $T_{d}$ \\
\hline ZN & 4.0588 & 9.2500 & 2.3125 \\
ISE & 4.4635 & 30.5449 & 2.3231 \\
IMC $\left(T_{f}=1.3\right)$ & 3.6177 & 22.4300 & 2.1824 \\
IFT & 3.6717 & 27.7222 & 2.1056 \\
\hline
\end{tabular}

PID parameters obtained with the 4 tuning methods for simulation 1 .

the IMC method, the presence of noise makes the estimation of an open loop model on the basis of a step response more difficult.

In order to evaluate the effect of a noise disturbance, we have performed simulations where the IFT parameters have been tuned in the presence of Gaussian noise acting on the output of the system. Two simulations have been performed; in each case the PID parameters have been tuned by the IFT method, with the same mask strategy as above.

(1) In the first simulation, a white Gaussian noise, with standard deviation $\sigma=0.05$, has been added to the output of the closed loop system during all iterations. The PID parameters obtained under those conditions by the IFT tuning method are now $K=2.7272, T_{i}=28.3081$ and $T_{d}=1.3366$; compare with the values given in Table 2 under noise-free conditions.

(2) In the second simulation colored Gaussian noise has been added to the output. The colored noise was obtained by passing white Gaussian noise, with standard deviation $\sigma=0.05$, through a low-pass filter $H(z)=$ $\frac{1}{1-0.8 z^{-1}}$. The PID parameters obtained under those conditions by the IFT tuning method are now $K=2.5778, T_{i}=30.2147$ and $T_{d}=2.5043$.

The closed loop responses with the corresponding IFT-tuned regulators, under the same noise conditions, are shown in Figure 3 (for the case of white Gaussian noise) and in Figure 4 (for the case of colored noise). In each case, the closed loop responses are compared with the closed loop response obtained with the IMC controller of Table 1 under the same noise conditions. These figures show the clear advantage of having a PID controller tuning method that can take account of the presence of noise disturbances: the IFT controller clearly outperforms the IMC controller in the presence of either white or colored noise. 

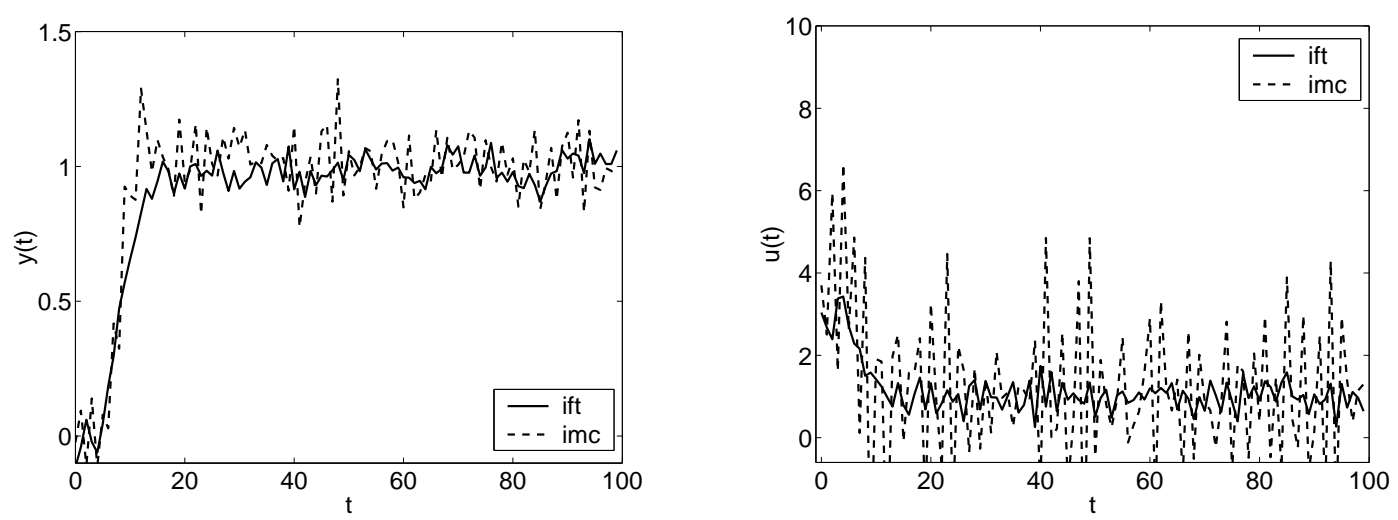

Fig. 3. Step responses (left) and corresponding input signals (right) for the closed loop systems with $G_{1}(s)$ and white noise perturbation, for the IFT and IMC controller.
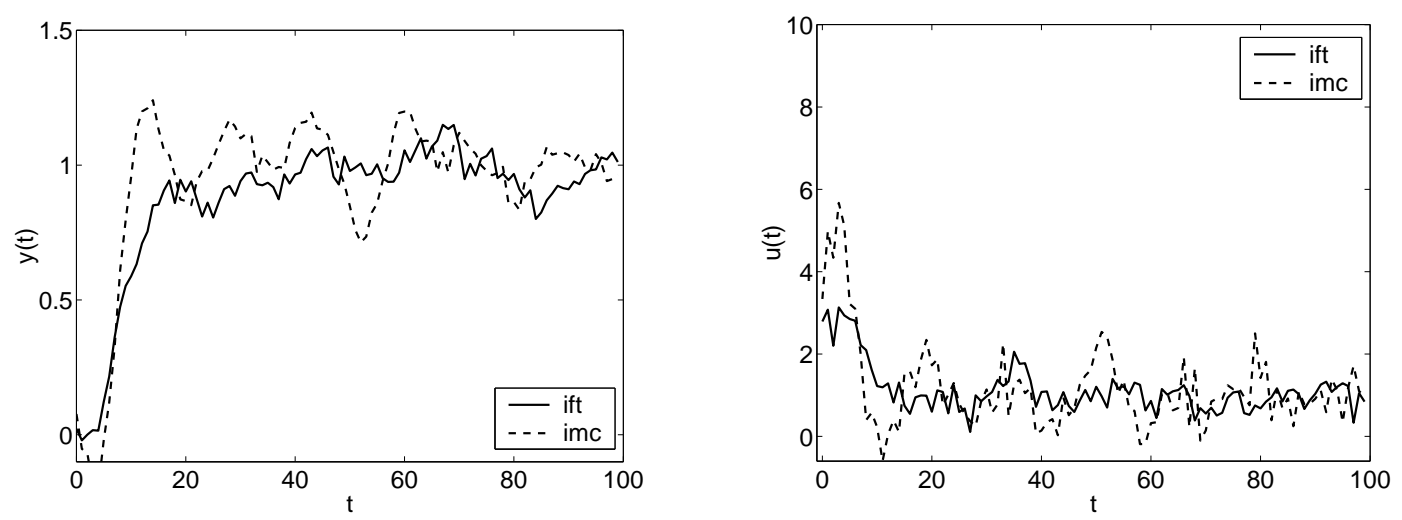

Fig. 4. Step responses (left) and corresponding input signals (right) for the closed loop systems with $G_{1}(s)$ and colored noise perturbation, for the IFT and IMC controller.

\subsection{Simulation 2}

The simulated system is the same as in the first simulation but with a delay that is four times longer:

$$
G_{2}(s)=\frac{1}{1+20 s} e^{-20 s}
$$

The closed loop step responses obtained by the four PID tuning methods for system $G_{2}$ are shown in Figure 5 . For the IFT method, $t_{0}$ has been decreased 
from 150 secs to 50 secs in steps of 25 secs, while for the IMC method the design parameter was optimized at $T_{f}=14$.
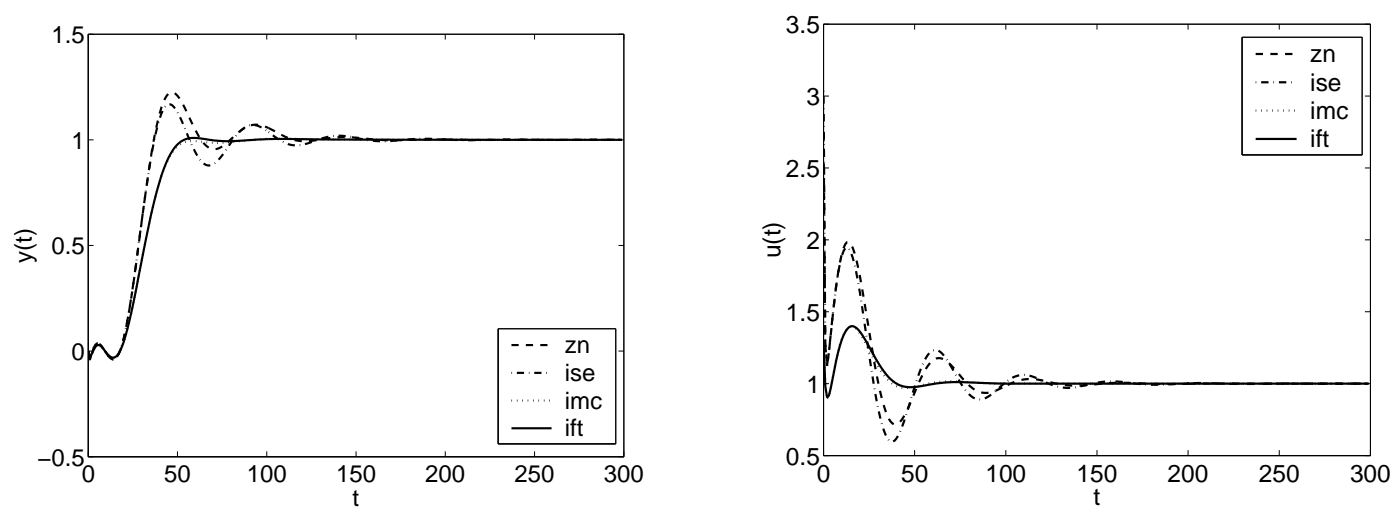

Fig. 5. Step responses (left) and corresponding input signals (right) for the closed loop systems with $G_{2}(s)$ and the PID controllers tuned with the four different methods.

With this longer delay in the system, the responses of the IFT controller and the IMC controller with an optimized $T_{f}$ are almost indistinguishable. Their superiority over the Ziegler-Nichols and ISE regulators is even more striking. The IFT and IMC controllers exhibit almost no overshoot, with a control action that is significantly smoother than for the other two competitors.

The PID parameters produced by the four different tuning methods are given in Table 2.

Table 2

\begin{tabular}{|l||c|c|c|}
\hline Tuning method & $K$ & $T_{i}$ & $T_{d}$ \\
\hline ZN & 1.3294 & 30.95 & 7.7375 \\
ISE & 1.3611 & 36.4409 & 8.1072 \\
IMC $\left(T_{f}=14\right)$ & 0.9351 & 30.5400 & 6.4797 \\
IFT & 0.9303 & 30.0593 & 6.0553 \\
\hline
\end{tabular}

PID parameters obtained with the 4 tuning methods for simulation 2 .

\subsection{Simulation 3}

The simulated system has a single pole of order 8 :

$$
G_{3}(s)=\frac{1}{(1+10 s)^{8}} .
$$


The closed loop step responses obtained by the four PID tuning methods for system $G_{3}$ are shown in Figure 6 . For the IFT method, $t_{0}$ has been decreased from 280 secs to 130 secs in steps of 30 secs, while $T_{f}$ was optimized at 42 for the IMC method.
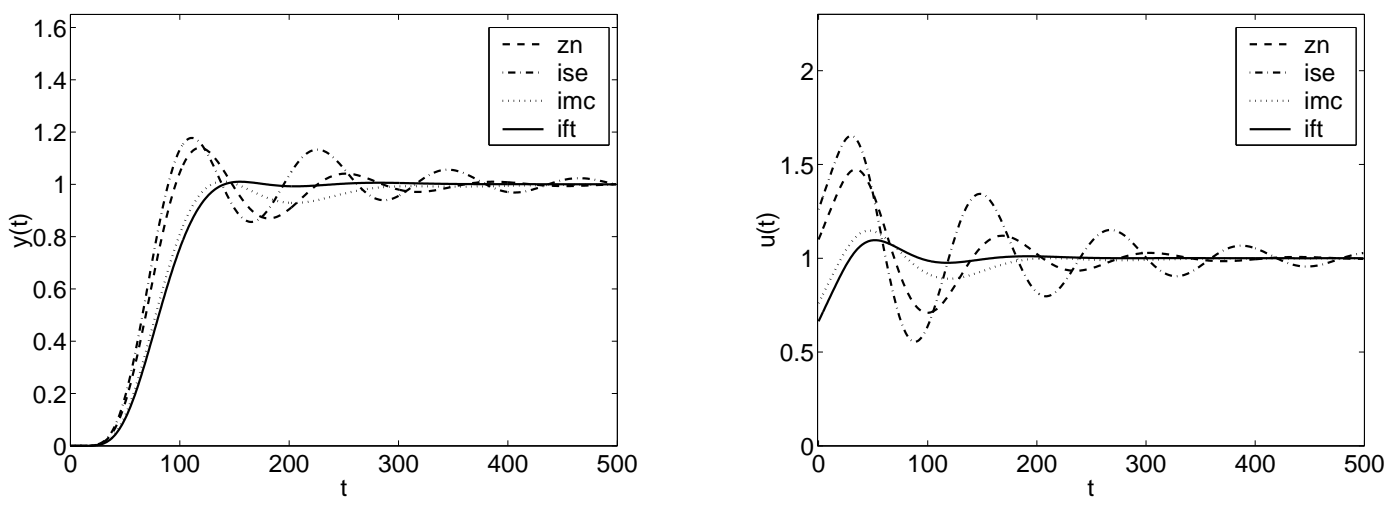

Fig. 6. Step responses (left) and corresponding input signals (right) for the closed loop systems with $G_{3}(s)$ and the PID controllers tuned with the four different methods.

The settling time of the IFT controller is significantly shorter than that achieved by the three other schemes, while the control action of IFT and IMC is much smaller than that of ZN and ISE.

The PID parameters produced by the four different tuning methods are given in Table 3.

Table 3

\begin{tabular}{|l||c|c|c|}
\hline Tuning method & $K$ & $T_{i}$ & $T_{d}$ \\
\hline ZN & 1.1000 & 75.9000 & 18.9750 \\
ISE & 1.2606 & 74.1044 & 26.3030 \\
IMC $\left(T_{f}=42\right)$ & 0.7604 & 64.6850 & 14.3655 \\
IFT & 0.6641 & 53.9791 & 18.2139 \\
\hline
\end{tabular}

PID parameters obtained with the 4 tuning methods for simulation 3 .

\subsection{Simulation 4}

The simulated system has a nonminimum phase zero:

$$
G_{4}(s)=\frac{1-5 s}{(1+10 s)(1+20 s)} .
$$

The closed loop step responses obtained by the four PID tuning methods for system $G_{4}$ are shown in Figure 7 . For the IFT method, $t_{0}$ has been decreased 
from 110 secs to 30 secs in steps of 20 secs, while $T_{f}$ was optimized at 0.2 for the IMC method.
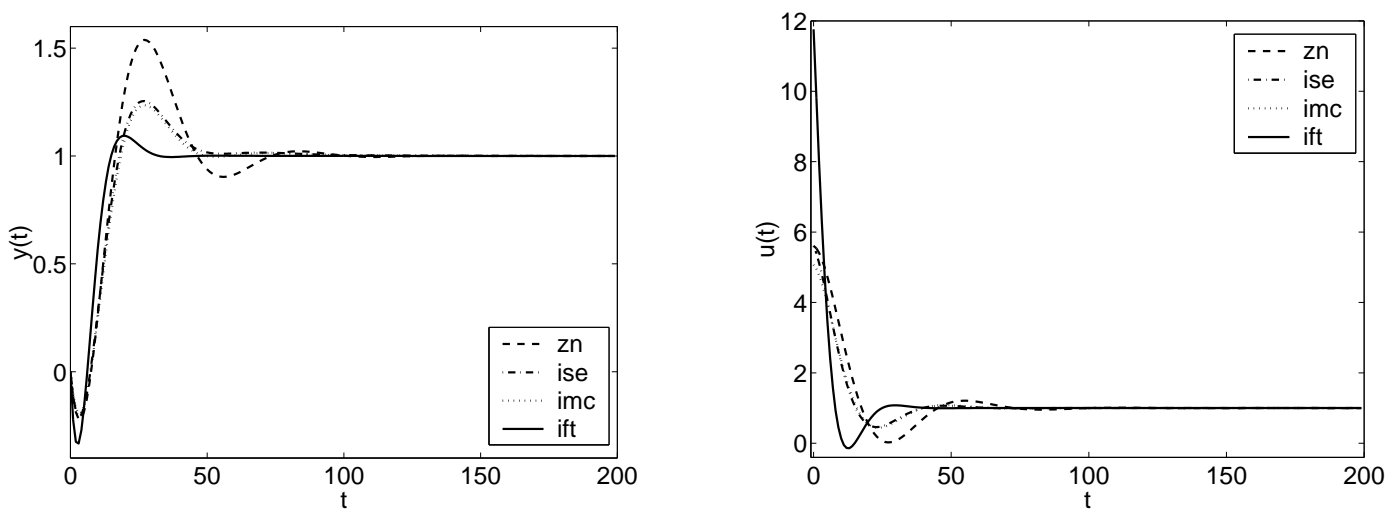

Fig. 7. Step responses (left) and corresponding input signals (right) for the closed loop systems with $G_{4}(s)$ and the PID controllers tuned with the four different methods.

We observe that IFT produces by far the best closed loop step response. However, this is achieved through application of a control action that is initially much larger than with the other methods. In order to reduce this large control action, we have recomputed the PID parameters using the IFT method, but with a penalty added on the control effort. Thus the following criterion has been adopted:

$$
J(\boldsymbol{\rho})=\mathrm{E}\left\{\sum_{t=t_{0}}^{N}\left(y_{t}(\boldsymbol{\rho})-y_{t}^{d}\right)^{2}+\lambda \sum_{t=0}^{N}\left(u_{t}(\boldsymbol{\rho})\right)^{2}\right\},
$$

where $\lambda=1 \cdot 10^{-7}$. The width of the mask, $t_{0}$, was again decreased from 110 secs to 30 secs in steps of 20 secs. The results are shown in Figure 8.

Thanks to the penalty on the control effort in the IFT criterion, the control action is now never larger than that obtained with the classical controllers. In addition, the control signal settles faster than with the other controllers. Perhaps somewhat surprisingly, the closed loop step response has also improved with respect to the IFT controller obtained without penalty on the control action: the output settles to its new steady-state value in a minimum time and without any overshoot. Finally, note that better results could have possibly been obtained with the IMC method if a more complex model structure had been adopted than (9); however, this would require more modeling work.

The PID parameters produced by the four different tuning methods are given in Table 4. 

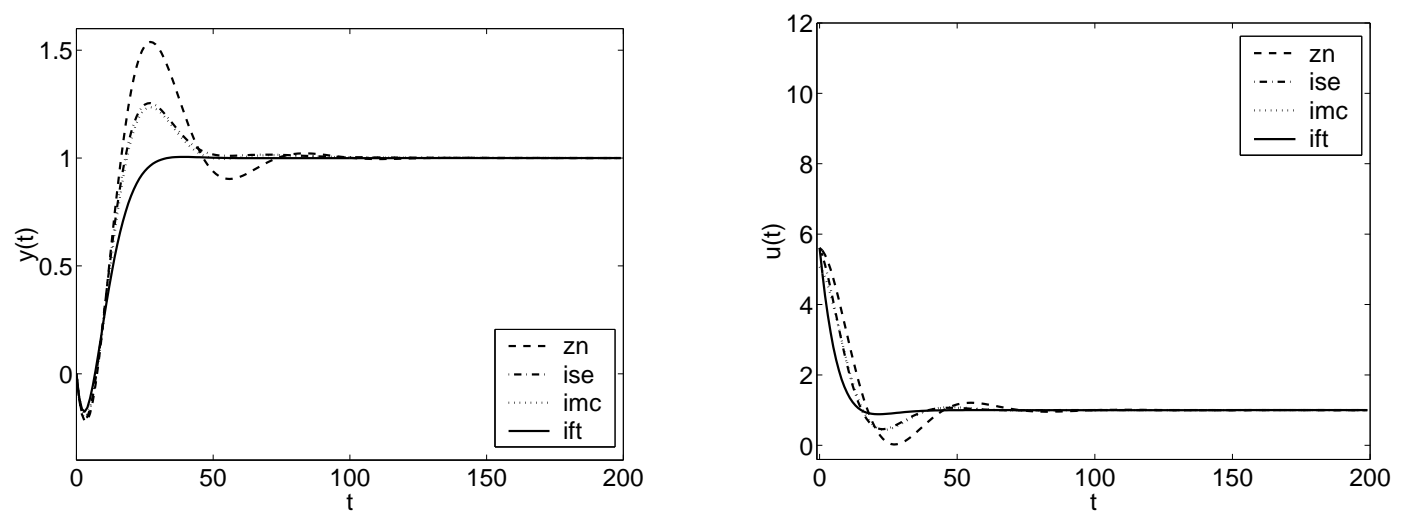

Fig. 8. Step responses (left) and corresponding input signals (right) for the closed loop systems with $G_{4}(s)$ and the PID controllers tuned with the three classical methods, and with IFT with weighting $\lambda=1 \cdot 10^{-7}$.

Table 4

\begin{tabular}{|l||c|c|c|}
\hline Tuning method & $K$ & $T_{i}$ & $T_{d}$ \\
\hline ZN & 3.5294 & 16.8000 & 4.2000 \\
ISE & 3.5300 & 28.7450 & 4.200 \\
IMC $\left(T_{f}=0.2\right)$ & 3.3926 & 31.5850 & 3.8981 \\
IFT $\left(\lambda=1 \cdot 10^{-7}\right)$ & 3.0279 & 46.3178 & 6.0793 \\
\hline
\end{tabular}

PID parameters obtained with the 4 tuning methods for simulation 4 .

\section{Robustness to model errors}

One of the important properties of any controller tuning method is its robustness to model errors. In order to evaluate the robustness of IFT and to compare it with that of the three classical tuning methods, we have evaluated the closed loop responses achieved by the four PID controllers listed in Table 4 when these controllers are applied to a model that is slightly different from the model $G_{4}(s)$ with which these controllers were computed.

In Figure 9, we present the closed loop outputs and control inputs achieved by these four controllers when applied to the following perturbed system, in which the steady state gain of $G_{4}(s)$ has been increased by $50 \%$ :

$$
G_{4 a}(s)=\frac{1.5(1-5 s)}{(1+10 s)(1+20 s)}
$$

In Figure 10, we present the closed loop outputs and control inputs achieved by these controllers when applied to the following perturbed system, in which 

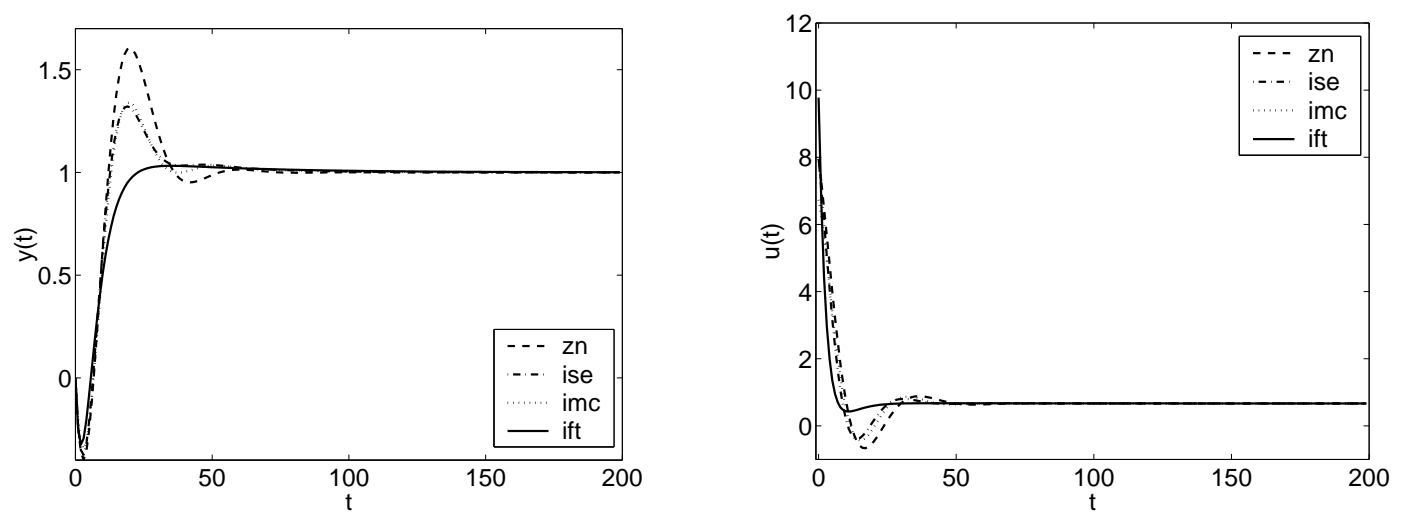

Fig. 9. Step responses (left) and corresponding input signals (right) achieved by the four PID controllers of Table 4 when applied to the perturbed system $G_{4 a}(s)$.

the slow time constant of $G_{4}(s)$ has been changed from 20 to 25 :

$$
G_{4 b}(s)=\frac{1-5 s}{(1+10 s)(1+25 s)} .
$$
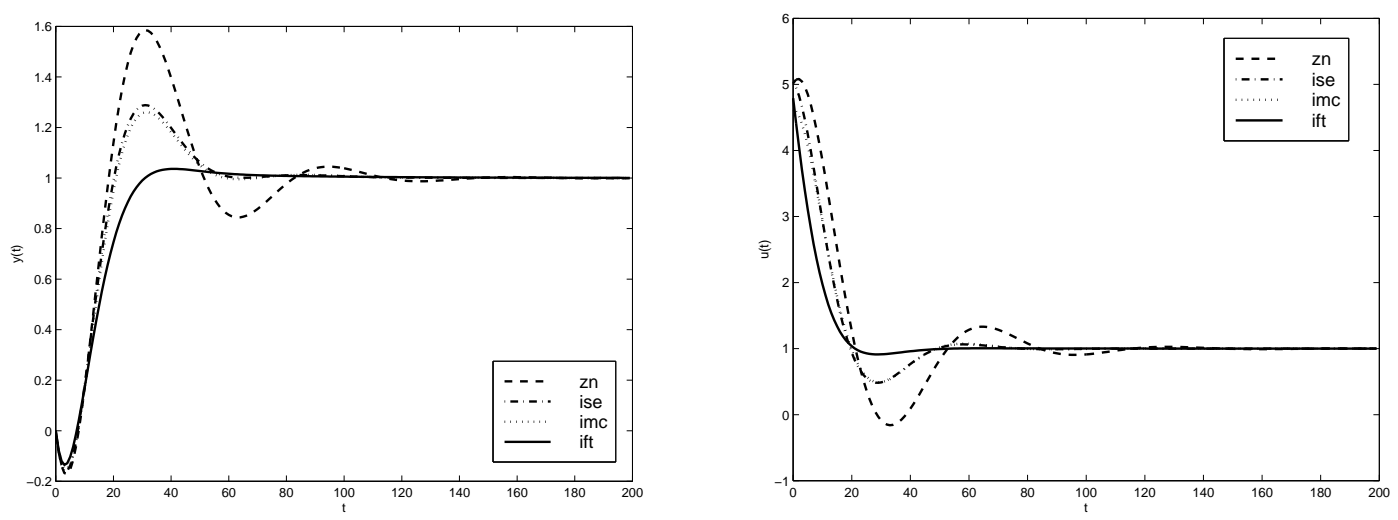

Fig. 10. Step responses (left) and corresponding input signals (right) achieved by the four PID controllers of Table 4 when applied to the perturbed system $G_{4 b}(s)$.

In Figure 11, we present the closed loop outputs and control inputs achieved by these controllers when applied to the following perturbed system, in which a delay of 3 seconds has been added to $G_{4}(s)$ :

$$
G_{4 c}(s)=\frac{(1-5 s)}{(1+10 s)(1+20 s)} e^{-3 s} .
$$

All four controllers are rather robust to these modeling errors, except when the mismatch is on the delay, which causes an increase in the settling time. However, this increase is rather more moderate with the IFT controller. 

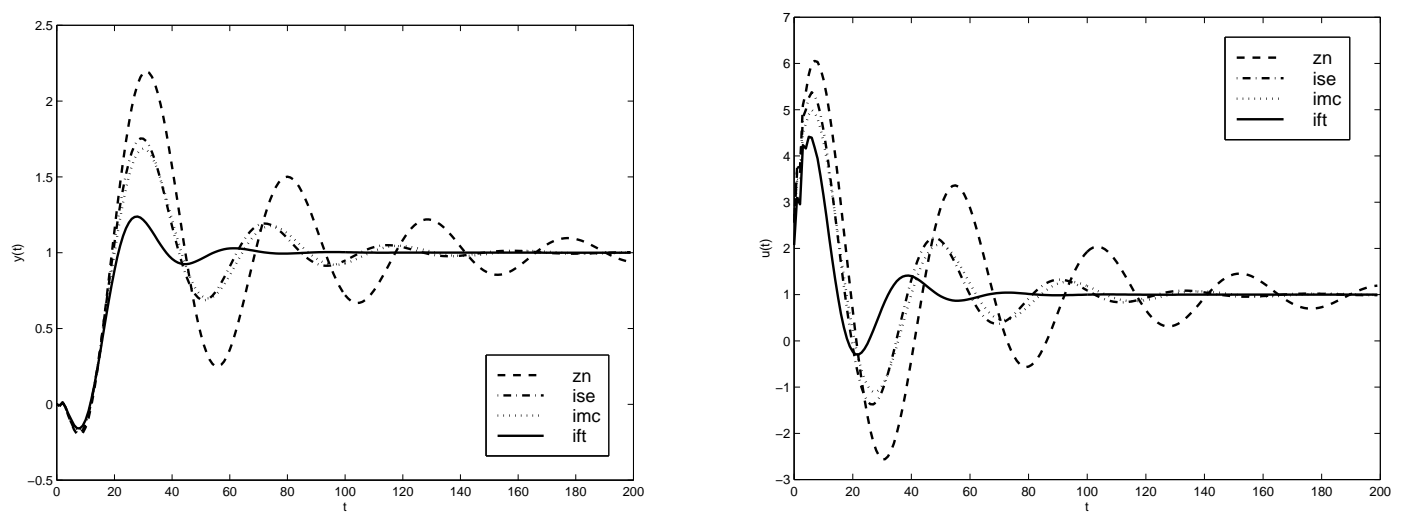

Fig. 11. Step responses (left) and corresponding input signals (right) achieved by the four PID controllers of Table 4 when applied to the perturbed system $G_{4 c}(s)$.

\section{Comments and observations}

In performing these and other simulations, we have made a number of recurrent observations.

Very few iterations are needed for the IFT method when the sampling interval is large. More iterations seem to be needed for smaller sampling intervals, but the result will be about the same.

It is always advisable to start the IFT iterations with a large mask and to reduce the size of the mask in successive steps. The final mask size is roughly equal to the rise time that can be achieved with minimal or no overshoot. However, even if one knew a priori which rise time can be achieved without significant overshoot, it would be wrong to use a mask of corresponding length straight away. This has been illustrated in the following example, first presented in (Lequin et al., 1999).

Consider the plant $y(t)=G(s) u(t)+H(s) e(t)$, with

$$
G(s)=\frac{1}{s^{2}+0.1 s+1}, \quad H(s)=\frac{1}{s+1},
$$

where $e(t)$ is white noise with variance $\sigma^{2}=0.0025$. One wishes to tune a PID controller in order to achieve a settling time of 20 seconds for the closed loop system. The initial PID parameter values are taken as $K=0.025, T_{i}=2$ and $T_{d}=1$. This yields the very sluggish response shown in Figure 12 .

The application of the IFT criterion with a fixed mask of width $t_{0}=20$ seconds with these initial parameters leads to the closed loop response of Figure 13. 


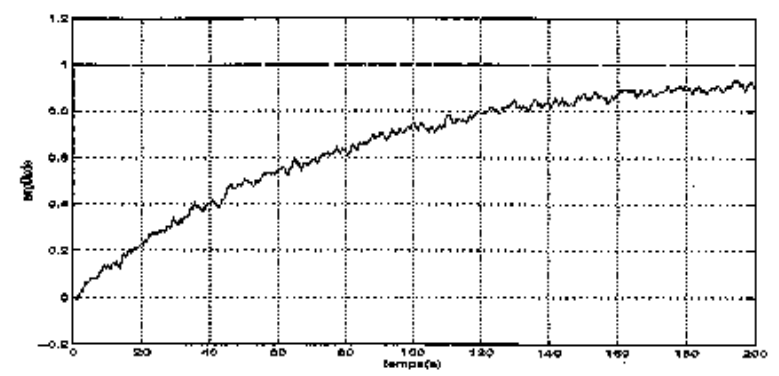

Fig. 12. Closed loop step response with initial PID parameters

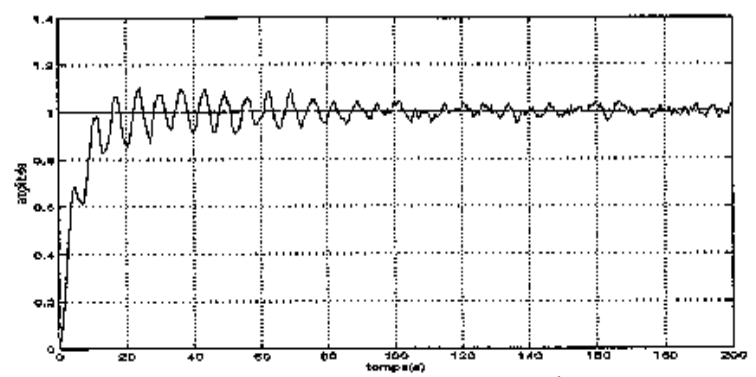

Fig. 13. Optimal closed loop step response obtained with the IFT criterion using a mask of length 20

A mask of decreasing length was then used, with an initial length of 80 seconds, and with the same initial parameters again. At every iteration of the IFT scheme, the length of the mask was decreased by 20 seconds, until a mask of length 20 was reached. This led to the closed loop response shown in Figure 13.

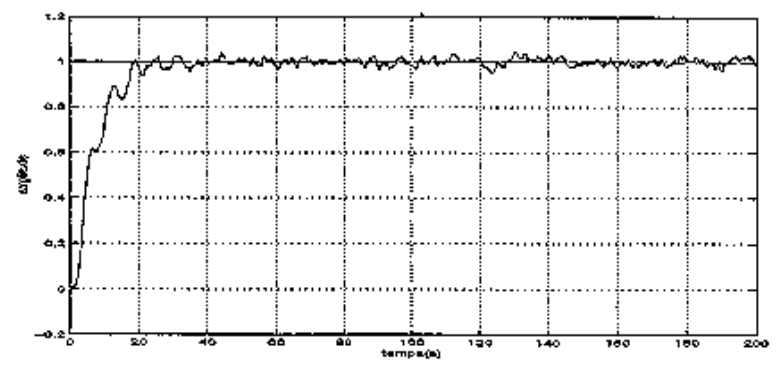

Fig. 14. Optimal closed loop step response obtained with the IFT criterion using masks of decreasing length

Observe the dramatic improvement of the response of Figure 13 vis-à-vis the response of Figure 12, even though in both cases the final criterion is identical (i.e. the criterion $J_{m}(\rho)$ of (4) with a mask of length $t_{0}=20$ ), and even though the starting values used for $\rho_{0}$ were identical in both simulations. The improvement is due to the use of masks of decreasing lengths in the last simulation, leading to a sequence of cost criteria (rather than a one-shot criterion), and to a different sequence of $\rho_{i}$ parameter vectors than resulted with the direct use of a mask of length $t_{0}=20$. Even though the final criteria 
are identical, the minima that are reached by the two approaches are quite different. Our interpretation is as follows. In the case when the final criterion (with a mask length of $t_{0}=20$ ) is adopted from the start, the initial parameter vector $\rho_{0}$ is very far from the optimal parameter $\rho^{\star}$ that yields the global minimum of the cost criterion. As a result, there is a great risk that the sequence of parameters $\rho_{i}$ produced by the iterative method gets stuck into a local minimum. In the case where the mask length is progressively reduced, one minimizes a sequence of criteria that are not too different from one another. For each of these minimization problems, the initial parameter vector is not too distant from the global minimum of the criterion that is minimized, and hence the risks of getting stuck in a local minimum are significantly reduced.

\section{Conclusions}

We have developed a simple and efficient method to tune simple controllers, such as PID controllers, in order to achieve minimum settling time with no overshoot or with minimal overshoot. The scheme consists in computing the controller parameters iteratively using a variant of the IFT scheme in which no control error weighting is applied during the transient phase of the closed loop step response. The width of this zero-weight window is initially taken much larger than the settling time that one expects to achieve. This leads to an initial closed loop response that is very sluggish. The window width is then progressively reduced until oscillations start occurring.

We have illustrated the performance of this optimal tuning method for the tuning of PID controllers, through four simulation examples representative of different situations, including a long time delay, or a non-minimum phase zero. On these four examples, we have compared it with three classical PID tuning methods: the new variant of the IFT scheme performs significantly better than the ZN and ISE controllers, and is comparable with the IMC controller. It has the advantage over IMC that it is not necessary to open the loop, and that a noise rejection objective is built in the design process. However, note that the IFT method with masks presented here requires a starting value for the PID parameters. Thus, it must be seen as complementary to one-shot PID design methods. It would be interesting to use the IFT tuning method presented in this paper as a second step (for optimization purposes) of the recently developed Virtual Reference Feedback Tuning (VRFT) method (Campi et al., 2000). 


\section{Acknowledgements}

M. Gevers acknowledges the Belgian Programme on Inter-university Poles of Attraction, initiated by the Belgian State, Prime Minister's Office for Science, Technology and Culture. The scientific responsibility rests with its authors. M. Mossberg and M. Gevers acknowledge the financial support provided by the European Commission. This support is provided via the Program Training and Mobility of Researchers (TMR) and Project System Identification (ERB FMRX CT98 0206) to the European Research Network System Identification (ERNSI).

\section{References}

Åström, K. J. (1970). Introduction to Stochastic Control Theory. Academic Press. New York, NY.

Åström, K.J. and B. Wittenmark (1997). Computer Controlled Systems: Theory and Design - Third Edition. Prentice-Hall. Upper Saddle River, New Jersey.

Åström, K.J. and T. Hägglund (1995). PID Controllers: Theory, Design and Tuning, 2nd Edition. Instrument Society of America. North Carolina, USA.

Campi, M.C., A. Lecchini and S.M. Savaresi (2000). Virtual Reference Feedback Tuning (VRFT): a new direct approach to the design of feedback controllers. In: Proc. 39th IEEE Conference on Decision and Control. Sydney, Australia. pp. 623-628.

Hjalmarsson, H., M. Gevers, S. Gunnarsson and O. Lequin (1998). Iterative Feedback Tuning: theory and applications. IEEE Control Systems Magazine 18, 26-41.

Hjalmarsson, H., S. Gunnarsson and M. Gevers (1994). A convergent iterative restricted complexity control design scheme. In: Proc. 33rd IEEE Conf. on Decision and Control. Orlando, Florida. pp. 1735-1740.

Hjalmarsson, H., S. Gunnarsson and M. Gevers (1995). Model-free tuning of a robust regulator for a flexible transmission system. European Journal of Control $\mathbf{1}(2), 148-156$.

Isaksson, A.J. and S.F. Graebe (1999). Analytical PID parameter expressions for higher order systems. Automatica 35(6), 1121-1130.

Kristiansson, B. and B. Lennartson (2000). From PI to $H_{\infty}$ control in a unified framework. In: Proc. 39th IEEE Conf. on Decision and Control. Sydney, Australia. 
Lequin, O. (1997). Optimal closed loop PID tuning in the process industry with the Iterative Feedback Tuning scheme. In: CD-ROM of European Control Conference, paper TH-A-H6. Brussels, Belgium.

Lequin, O., M. Gevers and L. Triest (1999). Optimizing the settling time with Iterative Feedback Tuning. In: Proc. 14th IFAC World Congress, paper I-3b08-3. Beijing, P.R. China. pp. 433-437.

Rivera, D.E. and M. Morari (1987). Control relevant model reduction problems for siso $H_{2}, H_{\infty}$ and $\mu$-controller synthesis. Int. Journal of Control 46(2), 505-527. 